\title{
Suplementação de glutamina em dietas elaboradas com ingredientes de origem vegetal e animal para pintos de corte
}

\author{
[Supplementation of glutamine in diets with ingredients from animal and \\ vegetable sources for broiler chicks] \\ K.L.A. Martinez ${ }^{1}$, N.S.M. Leandro ${ }^{2}$, M.B. Cafe $e^{2}$, J.H. Stringhini ${ }^{2}$, I.C.S. Araújo ${ }^{2}$, M.A. Andrade ${ }^{2}$ \\ ${ }^{1}$ Curso de Zootecnia - Universidade Federal de Goiás - Campus Jataí \\ ${ }^{2}$ Escola de Medicina Veterinária e Zootecnia - Universidade Federal de Goiás - Campus Goiânia
}

\begin{abstract}
RESUMO
Foi avaliado o efeito da suplementação da glutamina em dietas contendo ingredientes de origem animal sobre o desempenho e a integridade intestinal de pintos de corte, criados até 21 dias de idade. Os tratamentos constituíram-se de combinações entre tipos de dieta, com ingredientes de origem vegetal ou vegetal+animal e suplementação de glutamina $(0,0 ; 0,5 ; 1,0$ e $2,0 \%)$. O delineamento foi em blocos ao acaso em arranjo fatorial $2 \times 4$, tipos de ração x percentagem de glutamina, com cinco repetições e 12 pintos por unidade experimental. Não houve efeito da interação tipo de dieta versus suplementação de glutamina sobre o desempenho, e os tratamentos não influenciaram o desempenho de pintos de corte. Houve efeito quadrático da suplementação de glutamina sobre o coeficiente de digestibilidade da proteína bruta. A suplementação com glutamina aumentou altura de vilos e profundidade de cripta no duodeno. A utilização de produtos de origem animal em dietas para pintos na fase inicial não prejudica o desempenho, e a inclusão de glutamina melhora a integridade intestinal.
\end{abstract}

Palavras-chave: aminoácido, farinha de carne, farinha de pena, farinha de sangue, pintos de corte

\begin{abstract}
The effect of glutamine supplementation in diets formulated with animal by-products on the performance and integrity of the small intestine of broiler chicks up to 21 days of age was evaluated. The treatments were the combination of types of diets (only with ingredients from a vegetal source or vegetal plus animal source) and levels of glutamine (0.0; 0.5; 1.0 and 2.0\%). The experimental design was randomized blocks in a $2 \times 4$ factorial scheme (kinds of diets $x$ levels of glutamine), with five replicates and 12 birds per experimental unit. No interaction between diets and glutamine supplementation and treatment effects on the performance traits were observed during the chick starter phase. There was a quadratic effect of glutamine on the crude protein digestibility coefficient. Birds supplemented with glutamine diets showed higher villus height and crypt depth in the duodenum. Broiler diets formulated with animal ingredients have no effect on chick performance during the initial phase, and glutamine supplementation improved the small intestine integrity.
\end{abstract}

Keywords: aminoacid, blood meal, broiler chick, feather meal, meat meal

\section{INTRODUÇÃO}

A utilização de subprodutos de origem animal na formulação de rações pode ser considerada uma importante alternativa para preservação do meio ambiente, devido ao alto teor poluente que esses resíduos podem apresentar. Segundo Butolo (2002), no Brasil é permitida a utilização de farinhas de origem animal na alimentação de aves, suínos, peixes, cães e gatos, e o Ministério da Agricultura fiscaliza a indústria de reciclagem pela emissão de licenças para produção e registro dos produtos, além de realizar inspeções para verificar o cumprimento da legislação e a tolerância dos limites de segurança.

Recebido em 14 de junho de 2011

Aceito em 5 de setembro de 2012

*Autor para correspondência (corresponding author)

E-mail: mogyca@vet.ufg.br 
Nunes et al. (2006) relataram a necessidade de uma atenção especial quanto à qualidade desses produtos quando incluídos nas dietas em razão da variação que ocorre em sua composição nutricional e qualidade sanitária, que, por sua vez, pode comprometer o desempenho animal, por alterar a composição da microbiota intestinal.

O equilíbrio da microbiota é fundamental para assegurar a saúde intestinal (Macari e Maiorka, 2000). No entanto, alguns microrganismos interferem diretamente na integridade das células, podendo alterar a permeabilidade da membrana intestinal, modificando a capacidade de digestão e absorção de nutrientes, além de causar inflamações crônicas da mucosa (Loddi et al., 2006). Segundo Ito et al. (2004), a Escherichia coli, as Salmonellas, o Clostridium perfingens e a Lysteria monocytogenes são exemplos de microrganismos que podem produzir toxinas, alterar a fisiologia das células da mucosa intestinal, alterando a função celular ou mesmo causando sua destruição.

Frangos de corte infectados com microrganismos patogênicos apresentam formação de superóxido e radicais livres de óxido nítrico nos sítios de infecção (Allen, 1997). Segundo Wu et al. (1996), a glutamina pode eliminar esses radicais livres por ser precursor na síntese de glutationa, que é antioxidante. Ribeiro et al. (2004) afirmaram ainda que a glutamina pode contribuir para prevenção da translocação de bactérias e toxinas, auxiliando na manutenção da integridade intestinal. Em estudo com ratos desafiados com Escherichia coli, que receberam dieta com 2,0 ou $4,0 \%$ de glutamina, Inoue et al. (1993) observaram menor mortalidade para animais suplementados com o aminoácido. Do mesmo modo, Yi et al. (2005), ao avaliarem a inclusão de 2,0\% de glutamina na dieta de suínos, também desafiados com $E$ coli, constataram que o aminoácido favoreceu a manutenção da integridade intestinal nos animais desafiados.

De acordo com Butolo (2002), as Salmonellas e outros microrganismos são destruídos no momento do processamento dos resíduos de abatedouro, pois o material é submetido a temperaturas superiores a $100^{\circ} \mathrm{C}$. Contudo, pode haver contaminação após o processamento, manipulação e estocagem, em função da presença de microrganismos na área de processamento, do contato da matéria-prima com o produto acabado, presença de roedores e até mesmo pela utilização de embalagens impróprias ou contaminadas.

O objetivo deste trabalho foi avaliar o efeito da suplementação da glutamina em dietas com ingredientes de origem animal sobre $\mathrm{o}$ desempenho e integridade intestinal de frangos de corte na fase inicial.

\section{MATERIAL E MÉTODOS}

Para o experimento de desempenho e ensaio de metabolismo, foram usados 480 pintos de corte machos, de linhagem comercial, com um dia de idade. As aves foram alojadas em baterias metálicas, divididas em gaiolas medindo $0,80 \times 0,75 \times 0,25 \mathrm{~m}$, instaladas em galpão de alvenaria com $12,96 \times 2,96 \mathrm{~m}\left(38,36 \mathrm{~m}^{2}\right)$.

Os tratamentos constituíram de combinações entre dois tipos de dietas com ingredientes de origem vegetal ou origem vegetal+origem animal e suplementação de glutamina $-0,0 ; 0,5 ; 1,0$ e $2,0 \%$. O delineamento experimental utilizado foi em blocos ao acaso, com a altura da bateria considerada como bloco, seguindo arranjo fatorial $2 \times 4$ - dietas $\mathrm{x}$ glutamina na dieta, com cinco repetições e 12 aves por unidade experimental.

As aves criadas até 21 dias de idade receberam água e dieta à vontade, durante todo o período experimental. As dietas experimentais eram fareladas, $\mathrm{e}$ as que continham somente ingredientes de origem vegetal foram elaboradas à base de milho e farelo de soja. As dietas com os ingredientes de origem animal foram formuladas com milho, farelo de soja, farinha de carne, farinha de sangue e farinha de penas e vísceras (Tab. 1). Todas as dietas foram isonutritivas e formuladas com base nas exigências de aminoácidos totais, para atender às exigências nutricionais de acordo com Rostagno et al. (2005), exceto para a glutamina, cujas percentagens de inclusão foram obtidas com a suplementação de glutamina na forma de aminoácido sintético L-Glutamina em substituição ao amido de mandioca. As dietas não continham agentes anticoccidianos ou promotores de crescimento. 
Tabela 1. Composição percentual e calculada das dietas basais, com ingredientes de origem vegetal (RV) ou ingredientes de origem vegetal e animal $(\mathrm{RV}+\mathrm{A})$

\begin{tabular}{|c|c|c|}
\hline Ingrediente (\%) & RV & $\mathrm{RV}+\mathrm{A}$ \\
\hline Milho & 53,130 & 60,501 \\
\hline Farelo de soja 44 & 36,570 & 26,193 \\
\hline Farinha de carne e ossos 45 & 0,00 & 4,285 \\
\hline Farinha de penas e vísceras & 0,00 & 2,00 \\
\hline Farinha de sangue & 0,00 & 1,00 \\
\hline Óleo de soja & 3,971 & 2,00 \\
\hline Cloreto de sódio & 0,496 & 0,279 \\
\hline Calcário & 0,931 & 0,274 \\
\hline Fosfato bicálcico & 1,841 & 0,200 \\
\hline Pré-mistura Min. e Vit. ${ }^{1}$ & 0,400 & 0,400 \\
\hline DL-Metionina & 0,316 & 0,292 \\
\hline L-Lisina & 0,229 & 0,320 \\
\hline L-Treonina & 0,116 & 0,106 \\
\hline Bicarbonato de Sódio & 0,00 & 0,200 \\
\hline Amido de mandioca $^{2}$ & 2,00 & 2,00 \\
\hline Total & 100,00 & $\mathbf{1 0 0 , 0 0}$ \\
\hline \multicolumn{3}{|l|}{ Composição calculada: } \\
\hline Energia metabolizável $(\mathrm{kcal} / \mathrm{kg})$ & 3000 & 3010 \\
\hline Proteína bruta $(\%)$ & 21,420 & 21,510 \\
\hline Lisina $(\%)$ & 1,365 & 1,365 \\
\hline Metionina (\%) & 0,638 & 0,596 \\
\hline Metionina+Cistina (\%) & 0,969 & 0,969 \\
\hline Cálcio (\%) & 0,905 & 0,906 \\
\hline Fósforo disponível (\%) & 0,449 & 0,449 \\
\hline
\end{tabular}

${ }^{1}$ Suplemento vitamínico e mineral (níveis de garantia por kg de produto): vit. A: 1.680.000UI; vit. D3: 400.000UI; vit. E: 3.500mg; vit. K: 360mg; vit. B1: 436,50mg; vit. B2: 1.200mg; vit. B6: $624 \mathrm{mg}$; vit. B12: 2.400mcg; ác. fólico: 200mg; ac. pantotênico; $3.120 \mathrm{mg}$; niacina; $8.400 \mathrm{mg}$; biotina; $10.000 \mathrm{mcg}$; colina: $78.300 \mathrm{mg}$; manganês: $18.750 \mathrm{ppm}$; zinco: $17.500 \mathrm{ppm}$; ferro: $12.500 \mathrm{ppm}$; cobre: $2.000 \mathrm{ppm}$; iodo: $187,50 \mathrm{ppm}$; selênio: $75 \mathrm{ppm}$; antioxidante: $2,5 \%$.

${ }^{2}$ Ingrediente utilizado em substituição à L-Glutamina.

O aquecimento de cada andar das baterias foi realizado com a instalação de lâmpadas incandescentes de $60 \mathrm{~W}$, sendo o aquecimento interno monitorado diariamente, e o manejo das cortinas, realizado para manter a temperatura interna do galpão adequada ao conforto das aves. Os comedouros e bebedouros utilizados foram do tipo linear, sendo limpos diariamente.

As características de desempenho avaliadas aos 7, 14 e 21 dias de idade foram: peso vivo, ganho de peso, consumo de dieta, conversão alimentar e viabilidade, sendo a conversão alimentar corrigida pelo peso das aves mortas.

Para a análise das características morfométricas do intestino delgado, foram sacrificadas cinco aves de cada tratamento aos 14 e 21 dias de idade, após jejum de seis horas. Segmentos de $3 \mathrm{~cm}$ do duodeno, jejuno e íleo foram colhidos de cada ave. Estes foram lavados em solução fisiológica, abertos pela sua borda mesentérica, estendidos pela túnica serosa e fixados em formol 10\%, por 24 horas. Posteriormente, o material foi lavado em álcool $70 \%$ e, em seguida, submetido à desidratação por tratamento com álcool em concentrações crescentes (70-100\%). As amostras foram diafanizadas em xilol e incluídas em parafina. Prepararam-se lâminas de cada segmento, realizando 10 cortes semisseriados, com sete micrômetros de espessura, corados com hematoxilina e eosina (HE), conforme metodologia descrtia por Luna (1968).

Após o procedimento de coloração, com o auxílio de um microscópio óptico acoplado a um sistema analisador de imagens Axio Vision 3.0 $\left(\right.$ Zeiss $^{\circledR}$ ), foram feitas medidas de altura e profundidade de criptas do duodeno, jejuno e íleo, sendo efetuadas 40 medidas de cada variável por animal. As medidas de altura do vilo 
foram tomadas a partir de sua região basal, coincidente com a porção superior das criptas, até seu ápice; e a profundidade de cripta, da sua base até a região de transição cripta:vilosidade, conforme descrito por Andrade (2005).

Nos períodos de 4 a 7 dias e de 12 a 15 dias de idade, foi realizado o ensaio de metabolismo, pelo método da colheita total de excretas. As coletas foram realizadas duas vezes ao dia, durante quatro dias, e foram acondicionadas em sacos plásticos identificados, congeladas para posteriores análises bromatológicas. As análises de proteína bruta, matéria seca e extrato etéreo das dietas e das excretas foram realizadas em duplicata, de acordo com metodologia descrita pela AOAC (Association..., 1990). A matéria seca a $65^{\circ} \mathrm{C}$ foi determinada em estufa de ventilação forçada à temperatura de $60^{\circ} \mathrm{C} \pm 5^{\circ} \mathrm{C}$ por 72 horas. A matéria seca a $105^{\circ} \mathrm{C}$ foi determinada em estufa regulada à temperatura de $105^{\circ} \mathrm{C}$ por oito horas. Para a determinação do extrato etéreo, foi empregado o método a quente, utilizando-se o extrator de Goldfisch da marca Tecnal. O nitrogênio total foi determinado pelo método micro Kjeldahl e o resultado, multiplicado pelo fator 6,25 para obtenção do percentual de proteína bruta.

Com os resultados das análises bromatológicas, foram calculados os coeficientes de digestibilidade (CD\%) da matéria seca (CDMS), do nitrogênio (CDN) e do extrato etéreo (CDEE), utilizando a fórmula:

$\mathrm{CD}(\%)=100 \mathrm{x}$ (Nutriente ingerido - Nutriente excretado) / Nutriente ingerido

A análise estatística das variáveis estudadas foi realizada por meio do programa GLM do $\mathrm{SAS}^{\circledR}($ Statistical..., 2000) e adotado o teste Tukey a 5\% de probabilidade para comparação das médias. Os dados foram submetidos à análise de regressão polinomial para as percentagens de inclusão de glutamina.

\section{RESULTADOS E DISCUSSÃO}

Não houve efeito da interação de tipo de dieta versus suplementação de glutamina $(\mathrm{P}>0,05)$ sobre o desempenho das aves no período de 1 a 7 dias de idade (Tab. 2). Para conversão alimentar, esse efeito foi quadrático $(\mathrm{P}<0,05)$, embora $\mathrm{o}$ coeficiente de determinação tenha sido baixo, segundo a equação $\mathrm{Y}=1,06+0,031 \mathrm{x}-0,02 \mathrm{x}^{2}$; $\mathrm{R}^{2}=0,30$. Os resultados indicam melhor conversão alimentar ao se utilizar $0,78 \%$ de glutamina na dieta. Resultados semelhantes foram obtidos por Nascimento (2010), sendo a adição de $1 \%$ de glutamina na dieta a que resultou em melhor conversão em relação à dieta sem a suplementação, durante a fase pré-inicial de criação.

Tabela 2. Desempenho de pintos de corte no período de 1 a 7 dias de idade, alimentados com diferentes tipos de dieta e suplementados com glutamina

\begin{tabular}{lccccc} 
Variável & $\begin{array}{c}\text { Peso } \\
\text { final }(\mathrm{g})\end{array}$ & $\begin{array}{c}\text { Ganho } \\
\text { de peso }(\mathrm{g})\end{array}$ & $\begin{array}{c}\text { Consumo dieta } \\
(\mathrm{g})\end{array}$ & $\begin{array}{c}\text { Conversão } \\
\text { alimentar }(\mathrm{g} / \mathrm{g})\end{array}$ & $\begin{array}{c}\text { Viabilidade } \\
(\%)\end{array}$ \\
\hline Glutamina, \% & & & & & \\
0,0 & 196,8 & 152,1 & $159,3 \mathrm{ab}$ & 1,059 & 98,3 \\
0,5 & 194,4 & 150,0 & $156,8 \mathrm{~b}$ & 1,055 & 98,3 \\
1,0 & 198,6 & 153,8 & $163,9 \mathrm{a}$ & 1,069 & 99,1 \\
2,0 & 201,6 & 156,8 & $160,8 \mathrm{ab}$ & 1,015 & 99,1 \\
\hline Tipo de dieta & & & & & \\
RV & 196,7 & 152,0 & 159,5 & $1,063 \mathrm{~b}$ & $97,9 \mathrm{~b}$ \\
RV+A & 199,1 & 154,4 & 160,9 & $1,037 \mathrm{a}$ & $99,5 \mathrm{a}$ \\
\hline Valor de P & & & & & \\
Glutamina & 0,10 & 0,13 & 0,01 & $<0,01$ & 0,65 \\
Dieta & 0,26 & 0,25 & 0,39 & 0,02 & 0,02 \\
Glutamina*Dieta & 0,36 & 0,45 & 0,22 & 0,88 & 2,34 \\
CV, \% & 4,7 & 6,0 & 4,3 & 4,5 & $\mathrm{~ns}$ \\
Regressão & $\mathrm{ns}$ & $\mathrm{ns}$ & $\mathrm{ns}$ & $0 \mathrm{uad}$ & 1 \\
\hline
\end{tabular}

$\mathrm{Na}$ mesma coluna, médias seguidas de letras diferentes diferem entre si pelo teste Tukey $(\mathrm{P} \leq 0,05)$.

$\mathrm{RV}=$ dieta elaborada somente com ingredientes de origem vegetal; $\mathrm{RV}+\mathrm{A}=$ dieta elaborada com ingredientes de origem vegetal $\mathrm{e}$ ingredientes de origem animal.

${ }^{1} \mathrm{Y}=1,06+0,031 \mathrm{x}-0,02 \mathrm{x}^{2}\left(\mathrm{R}^{2}=0,30\right)$. 
A suplementação de glutamina não influenciou os resultados de peso e ganho de peso e viabilidade das aves $(\mathrm{P}>0,05)$. Maiorka et al. (2000) não verificaram melhora no ganho de peso de frangos de corte aos sete dias de idade suplementados com $1 \%$ de glutamina na dieta. Sakamoto et al. (2009), em estudo para determinar a percentagem de adição de glutamina isolada ou associada ao ácido glutâmico na dieta, observaram que houve efeito da glutamina sobre o ganho de peso de frangos, o que aumentou conforme o acréscimo das fontes de glutamina nas dietas. Nascimento (2010) observou que o incremento da glutamina na dieta diminuiu a mortalidade de pintos de corte na fase inicial. Zavarize et al. (2011), ao suplementarem com glutamina a dieta de frangos de corte criados no sistema alternativo, observaram que a inclusão de $1 \%$ desse aminoácido aumentou o ganho de peso.

Com relação ao tipo de ingrediente utilizado na dieta, pode-se observar que a conversão alimentar foi melhor quando as dietas foram elaboradas com produtos de origem animal $(\mathrm{P}<0,05)$. Xavier et al. (2004) também observaram que frangos de corte alimentados com dieta contendo farinha de vísceras e penas apresentaram melhor conversão alimentar. Esses resultados podem ser explicados pela composição aminoacídica desse tipo de ingrediente que, segundo Brugalli (2002), pode apresentar perfil de aminoácidos similar àquele de que o animal necessita, sendo mais fácil atender às suas exigências, embora as dietas experimentais no presente estudo não tenham sido formuladas considerando o conteúdo de aminoácidos digestíveis dos ingredientes.

Este resultado sugere que as farinhas utilizadas nas dietas experimentais apresentavam excelente qualidade em relação ao processamento e contaminação microbiana. Contudo, deve-se considerar que, no presente experimento, as dietas elaboradas com produtos de origem animal continham somente $7,3 \%$ de sua composição com esse tipo de ingrediente, o que, provavelmente, não provocou uma situação de desafio para as aves. Algumas empresas têm adotado a inclusão de ingrediente de origem animal na dieta, e quando a qualidade sanitária desses produtos não é adequada, pode resultar em prejuízo à saúde intestinal das aves.

No período de 1 a 21 dias de idade (Tab. 3), não houve interação entre os fatores estudados $(\mathrm{P}>0,05)$, e não foi observado efeito do tipo de ingrediente utilizado na dieta sobre o desempenho ( $\mathrm{P}>0,05)$. Não houve efeito positivo da suplementação de glutamina, independentemente do tipo de dieta.

Tabela 3. Desempenho de frangos de corte no período de 1 a 21 dias de idade, alimentados com diferentes tipos de dieta e suplementados com glutamina

\begin{tabular}{lccccc}
\hline Variável & $\begin{array}{c}\text { Peso } \\
\text { final } \\
(\mathrm{g})\end{array}$ & $\begin{array}{c}\text { Ganho } \\
\text { de peso } \\
(\mathrm{g})\end{array}$ & $\begin{array}{c}\text { Consumo } \\
\text { dieta } \\
(\mathrm{g})\end{array}$ & $\begin{array}{c}\text { Conversão } \\
\text { alimentar } \\
(\mathrm{g} / \mathrm{g})\end{array}$ & $\begin{array}{c}\text { Viabilidade } \\
(\%)\end{array}$ \\
\hline Glutamina, \% & & & & & \\
0,0 & 981,20 & 936,46 & $1.184,02$ & 1,321 & 96,93 \\
0,5 & 957,16 & 912,77 & $1.157,86$ & 1,318 & 100 \\
1,0 & 964,18 & 919,37 & $1.184,75$ & 1,344 & 98,94 \\
2,0 & 953,88 & 909,01 & $1.179,57$ & 1,369 & 98,98 \\
\hline Dieta & & & & & \\
RV & 958,96 & 914,24 & $1.183,55$ & 1,359 & 98,96 \\
RV+A & 969,25 & 924,56 & $1.169,55$ & 1,317 & 98,46 \\
\hline Valor de P & & & & & \\
Glutamina & 0,83 & 0,84 & 0,51 & 0,74 & 0,26 \\
Dieta & 0,65 & 0,65 & 0,33 & 0,26 & 0,65 \\
Glutamina*Dieta & 0,50 & 0,51 & 0,57 & 0,35 & 0,47 \\
CV, \% & 7,5 & 7,8 & 3,8 & 8,7 & 3,5 \\
Regressão & $\mathrm{ns}$ & $\mathrm{ns}$ & $\mathrm{ns}$ & $\mathrm{ns}$ & $\mathrm{ns}$ \\
\hline Na & & &
\end{tabular}

Na mesma coluna, médias seguidas de letras diferentes diferem entre si pelo teste Tukey $(\mathrm{P} \leq 0,05)$.

$\mathrm{RV}=$ dieta elaborada somente com ingredientes de origem vegetal; $\mathrm{RV}+\mathrm{A}=$ dieta elaborada com ingredientes de origem vegetal e ingredientes de origem animal. 
Lopes (2008), ao estudar a adição de glutamina em dietas de frango de corte desafiados com Eimeria acervulina, não observou diferença para ganho de peso e peso vivo até 21 dias de idade. Contudo, observou melhor conversão alimentar para frangos alimentados com dietas suplementadas com glutamina e recomendou a adição de 1,4\% para essa fase de criação.

Com relação aos resultados do ensaio de metabolismo realizado no período de 5 a 7 dias de idade (Tab. 4), não foi observada interação entre tipo de dieta e suplementação de glutamina $(\mathrm{P}>0,05)$. A análise de regressão aplicada não foi significativa para os coeficientes de digestibilidade dos nutrientes na dieta.

Os tipos de ingredientes utilizados na dieta influenciaram o coeficiente de digestibilidade do extrato etéreo $(\mathrm{P}<0,05)$, com o melhor resultado quando as dietas foram elaboradas com ingredientes de origem animal. De acordo com Wiseman e Salvador (1991), Zollitsch et al. (1997) e Dvorin et al. (1998), a digestibilidade dos lipídeos está relacionada ao conteúdo de ácidos graxos, sendo que a digestibilidade das gorduras de origem animal é menor em relação às de origem vegetal, devido à menor quantidade de ácidos graxos insaturados na molécula.

Tabela 4. Coeficientes de digestibilidade da matéria seca (CDMS), da proteína bruta (CDPB) e do extrato etéreo (CDEE), obtidos nos períodos de 4 a 7 e de 12 a 15 dias de idade, em frangos alimentados com dietas suplementadas com glutamina

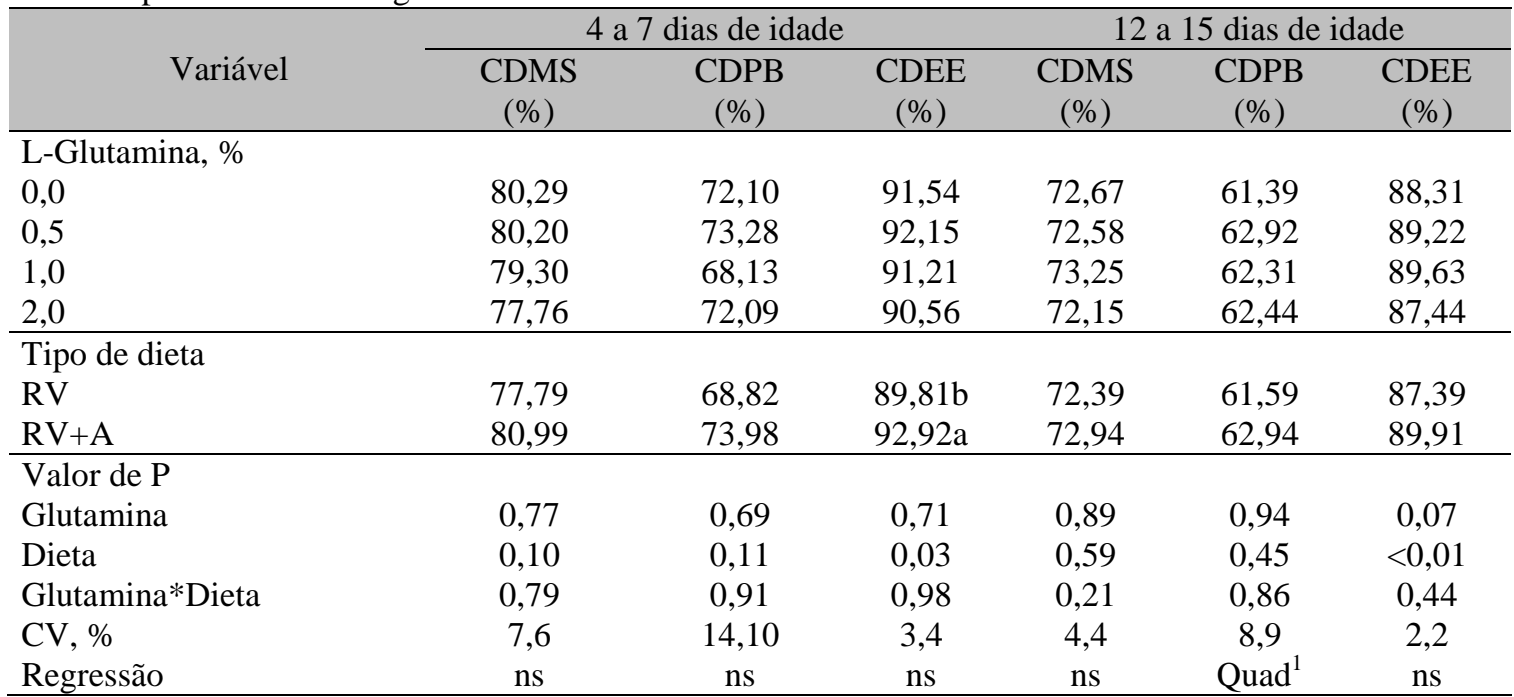

Na mesma coluna, médias seguidas de letras diferentes diferem entre si pelo teste Tukey $(\mathrm{P} \leq 0,05)$.

$\mathrm{RV}=$ dieta elaborada somente com ingredientes de origem vegetal; $\mathrm{RV}+\mathrm{A}=$ dieta elaborada com ingredientes de origem vegetal e ingredientes de origem animal.

${ }^{1} \mathrm{Y}=88,27+2,97 \mathrm{x}-1,69 \mathrm{x}^{2}\left(\mathrm{R}^{2}=0,13\right)$.

Não houve efeito da interação entre os fatores no ensaio de metabolismo realizado entre 12 a 15 dias de idade (Tab. 5). Os coeficientes de digestibilidade da matéria seca e do extrato etéreo não foram influenciados pela suplementação de glutamina nem pelos ingredientes utilizados na formulação das dietas $(\mathrm{P}>0,05)$. A regressão foi significativa para $\mathrm{o}$ coeficiente de digestibilidade da proteína bruta, resultando em uma equação quadrática, no entanto com $\mathrm{R}^{2}$ muito baixo $(\mathrm{Y}=88,27+2,97 \mathrm{x}$ $\left.-1,69 x^{2} ; R^{2}=0,13\right)$.
Houve interação para tipo de dieta versus suplementação de glutamina $(\mathrm{P}<0,05)$ para as características histomorfométricas do duodeno estudadas aos 14 dias de idade (Tab. 4). A suplementação com $1,0 \%$ de glutamina proporcionou maior altura de vilo em relação ao grupo não suplementado ou suplementado com $0,5 \%$ ou $2,0 \%$. A profundidade de cripta foi maior nas aves suplementadas com glutamina, em relação ao grupo não suplementado $(\mathrm{P}<0,05)$. Esses resultados sugerem que os subprodutos de origem animal exerceram efeito negativo no desenvolvimento da mucosa intestinal e que a 
glutamina pode ser benéfica quando for utilizado esse tipo de ingrediente nas rações. No entanto, pode-se observar que os resultados de desempenho na fase pré-inicial foram melhores para pintos alimentados com dietas com os produtos de origem animal e, portanto, pode-se sugerir que a melhor digestibilidade do extrato etéreo dessas dietas proporcionaram melhor conversão alimentar.

Tabela 5. Desdobramento das interações para altura do vilo, profundidade de cripta e relação vilo:cripta no duodeno de frangos aos 14 dias de idade

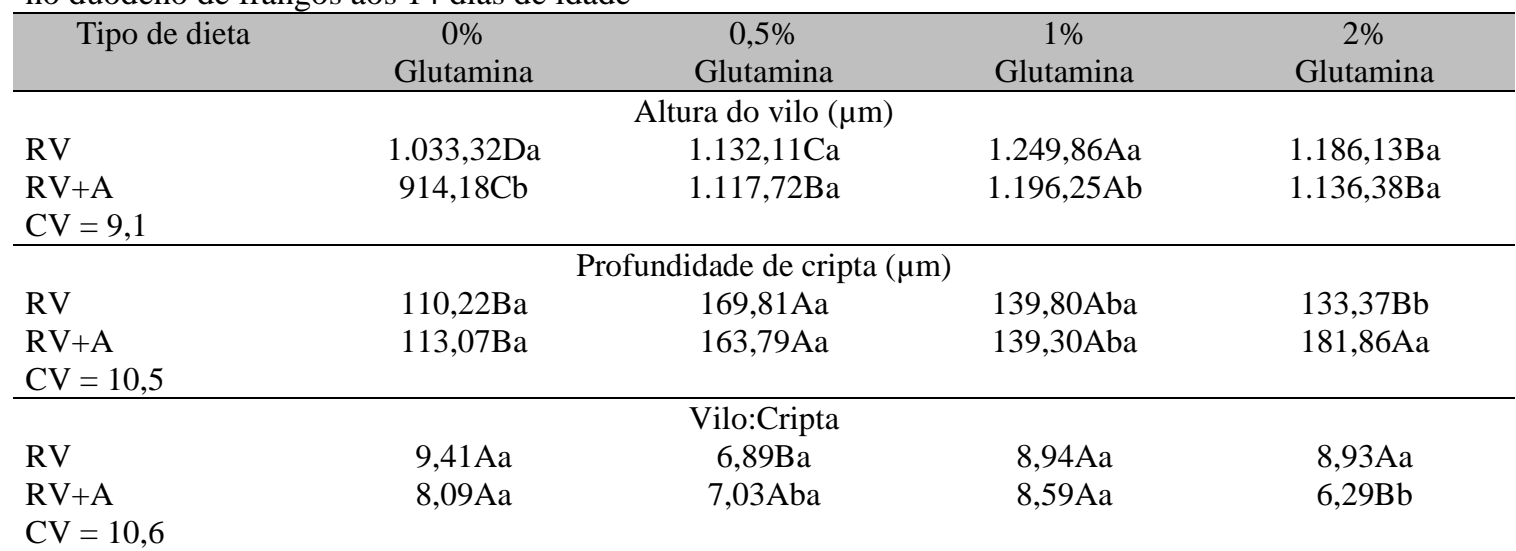

Médias seguidas por letras diferentes maiúsculas na linha ou minúsculas na coluna diferem entre si pelo teste Tukey $(\mathrm{P} \leq 0,05) . \mathrm{RV}=$ dieta elaborada somente com ingredientes de origem vegetal; $\mathrm{RV}+\mathrm{A}=$ dieta elaborada com ingredientes de origem vegetal e de origem animal.

Para as características do jejuno e íleo aos 14 dias de idade (Tab. 6), houve efeito quadrático $(\mathrm{P}<0,05)$ sobre todas as características estudadas, em função da suplementação de glutamina na dieta, indicando que 1,4 e $1,3 \%$ de glutamina promovem maior altura do vilo e profundidade de cripta, e relação vilo:cripta no jejuno. No íleo, os melhores resultados para altura do vilo, profundidade de cripta e relação vilo:cripta foram obtidos com 1,8,1,7 e 1,9\% de adição de glutamina, respectivamente.

Do mesmo modo, Sakamoto (2009) verificou que a suplementação da dieta na fase pré-inicial proporcionou melhora na morfometria duodenal de pintos de corte, alimentados com dietas suplementadas com glutamina isolada ou associada ao ácido glutâmico. Resultados semelhantes foram obtidos por Nascimento (2010), com suplementação de L-glutamina na ração.

A utilização de ingredientes de origem animal na dieta promoveu piores resultados de altura do vilo no jejuno e profundidade de cripta no íleo das aves aos 14 dias de idade $(\mathrm{P}<0,05)$. Houve interação para glutamina versus dieta para altura do vilo no íleo $(\mathrm{P}<0,05)$, indicando que, sem a suplementação, o melhor resultado obtido foi com dietas elaboradas somente com ingredientes de origem vetegal. A adição de 1,0\% de glutamina na dieta proporcionou melhor resultado, independentemente dos ingredientes utilizados na (Fig. 1).

Os resultados da análise histomorfométrica do intestino delgado aos 21 dias de idade estão apresentados na Tabela 6. Houve efeito quadrático de glutamina na dieta sobre as variáveis estudadas $(\mathrm{P}<0,05)$, exceto para altura de vilo e relação vilo:cripta no duodeno $(\mathrm{P}>0,05)$. Esses resultados indicam que, para se obter maior altura do vilo no duodeno, a inclusão de 1,9\% mostra-se mais adequada. Para o jejuno, as porcentagens de glutamina indicadas foram $1,2,0,7$ e $0,6 \%$, para altura do vilo, profundidade de cripta e relação vilo:cripta, respectivamente. Para as características do íleo, são indicadas 1,4 e $1,5 \%$ para altura do vilo e profundidade de cripta, e relação vilo:cripta, respectivamente. Sakamoto (2009), com a suplementação da glutamina isolada ou associada ao ácido glutâmico na dieta, em diferentes fases do programa alimentar, observou efeito da glutamina somente para profundidade da cripta do duodeno e para altura de vilo no íleo, sendo que a glutamina melhorou esses resultados para o período de 21 dias de idade. 
Martinez et al.

Tabela 6. Médias de altura do vilo (V), profundidade de cripta (C) e relação vilo:cripta (V:C) no jejuno e íleo de frangos aos 14 dias de idade

\begin{tabular}{|c|c|c|c|c|c|c|}
\hline \multirow[b]{2}{*}{ Variável } & \multicolumn{3}{|c|}{ Jejuno } & \multicolumn{3}{|c|}{ Íleo } \\
\hline & $\begin{array}{c}\mathrm{V} \\
(\mu \mathrm{m})\end{array}$ & $\begin{array}{c}\text { C } \\
(\mu \mathrm{m})\end{array}$ & $\mathrm{V}: \mathrm{C}$ & $\begin{array}{c}\mathrm{V} \\
(\mu \mathrm{m})\end{array}$ & $\begin{array}{c}\text { C } \\
(\mu \mathrm{m})\end{array}$ & $\mathrm{V}: \mathrm{C}$ \\
\hline \multicolumn{7}{|l|}{ Glutamina } \\
\hline $0,0 \%$ & 874 & 92 & 9,4 & 436 & 77 & 5,62 \\
\hline $0,05 \%$ & 1.004 & 108 & 9,2 & 450 & 75 & 6,02 \\
\hline $1,0 \%$ & 1.097 & 133 & 8,2 & 835 & 121 & 6,87 \\
\hline $2,0 \%$ & 1.072 & 121 & 8,8 & 795 & 112 & 7,05 \\
\hline \multicolumn{7}{|l|}{ Tipo de dieta } \\
\hline RV & $1.014 \mathrm{a}$ & 113 & 8,9 & $631 \mathrm{a}$ & $98 \mathrm{a}$ & 6,31 \\
\hline $\mathrm{RV}+\mathrm{A}$ & $1.009 \mathrm{~b}$ & 114 & 8,9 & $627 \mathrm{~b}$ & $95 b$ & 6,45 \\
\hline \multicolumn{7}{|l|}{ Valor P } \\
\hline Glutamina & $<0,01$ & $<0,01$ & $<0,01$ & $<0,01$ & $<0,01$ & $<0,01$ \\
\hline Dieta & $<0,01$ & 0,66 & 0,14 & $<0,01$ & 0,02 & 0,08 \\
\hline Glutamina*Dieta & 0,38 & 0,11 & 0,06 & $<0,01$ & 0,46 & 0,31 \\
\hline $\mathrm{CV}, \%$ & 2,1 & 2,1 & 2,1 & 3,2 & 3,4 & 4,3 \\
\hline Regressão & Quad $^{1}$ & Quad $^{2}$ & Quad $^{3}$ & Quad $^{4}$ & Quad $^{5}$ & Quad $^{6}$ \\
\hline
\end{tabular}

Na mesma coluna, médias seguidas de letras diferentes diferem entre si pelo teste Tukey $(\mathrm{P} \leq 0,05)$.

$\mathrm{RV}=$ dieta com ingredientes de origem vegetal; $\mathrm{RV}+\mathrm{A}=$ dieta com ingredientes de origem vegetal e ingredientes de origem animal. $\left.{ }^{1} \mathrm{Y}=871,35+340,65 \mathrm{x}-119,81 \mathrm{x}^{2(} \mathrm{R}^{2}=0,99\right) .{ }^{2} \mathrm{Y}=90,54+59,75 \mathrm{x}-21,86 \mathrm{x}^{2}\left(\mathrm{R}^{2}=0,90\right) .{ }^{3} \mathrm{Y}=9,55-$ $1,66 \mathrm{x}+0,63 \mathrm{x}^{2}\left(\mathrm{R}^{2}=0,62\right) \cdot{ }^{4} \mathrm{Y}=384,28+477,79 \mathrm{x}-131,66 \mathrm{x}^{2}\left(\mathrm{R}^{2}=0,76\right) .{ }^{5} \mathrm{Y}=70,98+52,07 \mathrm{x}-14,99 \mathrm{x}^{2}\left(\mathrm{R}^{2}=0,66\right) .{ }^{6} \mathrm{Y}=$ $5,54+1,58 \mathrm{x}-0,41 \mathrm{x}^{2}\left(\mathrm{R}^{2}=0,80\right)$.

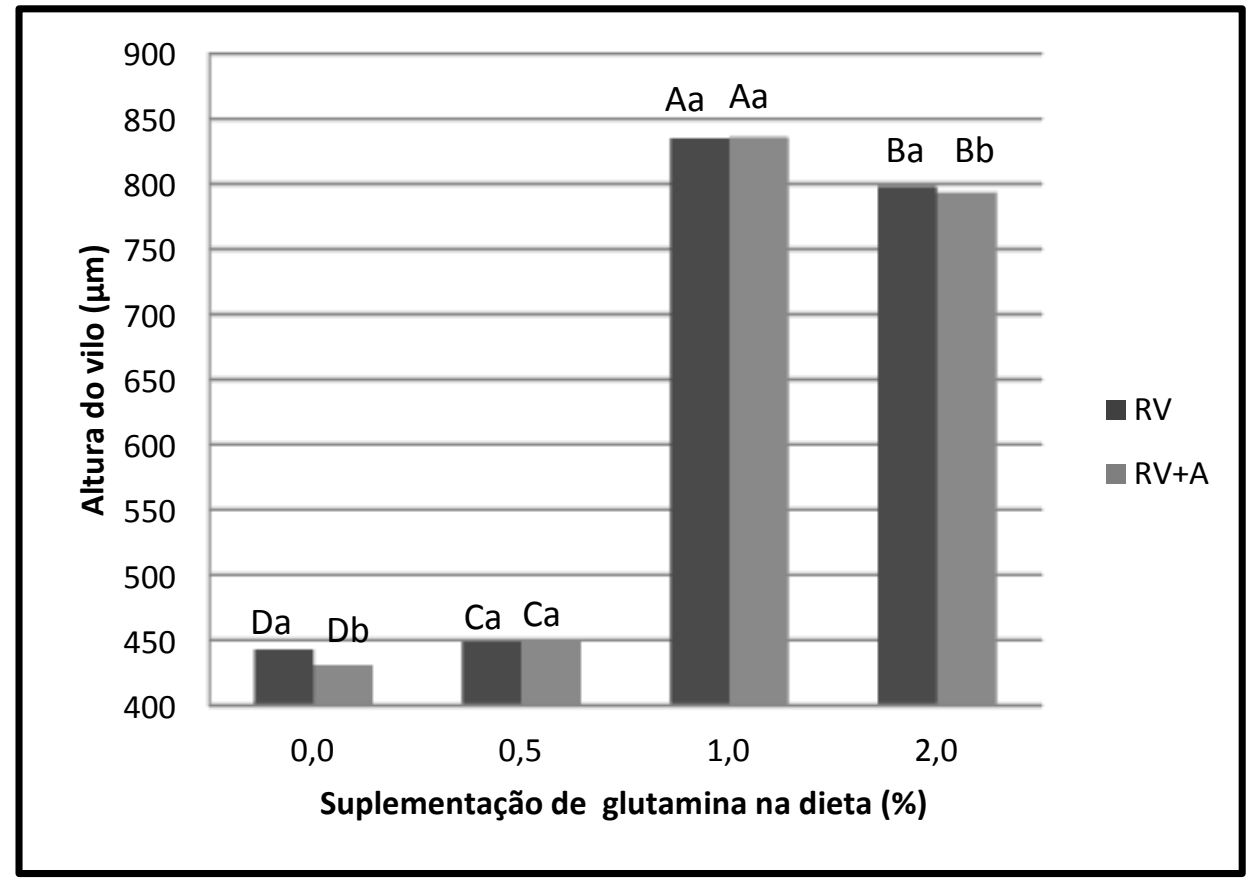

Figura 1. Altura do vilo no íleo de frangos de corte aos 14 dias, de acordo com o tipo de dieta e a suplementação de glutamina 
Suplementação de glutamina...

Tabela 6. Médias de altura do vilo (V), profundidade de cripta (C) e relação vilo:cripta (V:C) no intestino delgado de frangos aos 21 dias de idade

\begin{tabular}{|c|c|c|c|c|c|c|c|c|c|}
\hline \multirow[b]{2}{*}{ Variável } & \multicolumn{3}{|c|}{ Duodeno } & \multicolumn{3}{|c|}{ Jejuno } & \multicolumn{3}{|c|}{ Íleo } \\
\hline & $\begin{array}{c}\mathrm{V} \\
(\mu \mathrm{m})\end{array}$ & $\begin{array}{c}\mathrm{C} \\
(\mu \mathrm{m})\end{array}$ & $\mathrm{V}: \mathrm{C}$ & $\begin{array}{c}\mathrm{V} \\
(\mu \mathrm{m})\end{array}$ & $\begin{array}{c}\mathrm{C} \\
(\mu \mathrm{m})\end{array}$ & $\mathrm{V}: \mathrm{C}$ & $\begin{array}{c}\mathrm{V} \\
(\mu \mathrm{m})\end{array}$ & $\begin{array}{c}\mathrm{C} \\
(\mu \mathrm{m})\end{array}$ & $\mathrm{V}: \mathrm{C}$ \\
\hline Glutamina & & & & & & & & & \\
\hline $0,0 \%$ & 1.281 & $127 \mathrm{c}$ & $10,06 b$ & 791 & 112 & 7,02 & 593 & 88 & 6,68 \\
\hline $0,05 \%$ & 1.274 & $116 \mathrm{~d}$ & $10,94^{\mathrm{a}}$ & 785 & 106 & 7,39 & 581 & 83 & 6,96 \\
\hline $1,0 \%$ & 1.391 & $139 a$ & $9,95 b$ & 812 & 125 & 6,50 & 633 & 104 & 6,10 \\
\hline $2,0 \%$ & 1.379 & $136 b$ & $10,11 b$ & 795 & 88 & 9,00 & 611 & 95 & 6,44 \\
\hline Tipo de dieta & & & & & & & & & \\
\hline RV & 1.332 & 129 & 10,29 & 796 & 108 & 7,49 & $606 a$ & $94 a$ & $6,48 b$ \\
\hline $\mathrm{RV}+\mathrm{A}$ & 1.331 & 130 & 10,24 & 796 & 108 & 7,47 & $603 b$ & $91 b$ & $6,62 \mathrm{a}$ \\
\hline Valor P & & & & & & & & & \\
\hline Glutamina & $<0,01$ & $<0,01$ & $<0,01$ & $<0,01$ & $<0,01$ & $<0,01$ & $<0,01$ & $<0,01$ & $<0,01$ \\
\hline Dieta & 0,43 & 0,29 & 0,19 & 1,00 & 0,90 & 0,72 & $<0,01$ & $<0,01$ & $<0,01$ \\
\hline Gln*Dieta & 0,75 & 0,40 & 0,18 & 0,78 & 0,54 & 0,30 & 0,92 & 0,89 & 0,92 \\
\hline $\mathrm{CV}, \%$ & 3,2 & 4,7 & 2,9 & 2,5 & 2,3 & 2,4 & 2,6 & 3,4 & 3,5 \\
\hline Regressão & Quad $^{1}$ & ns & ns & Quad $^{2}$ & Quad $^{3}$ & Quad $^{4}$ & Quad $^{5}$ & Quad $^{6}$ & Quad $^{7}$ \\
\hline
\end{tabular}

Na mesma coluna, médias seguidas de letras diferentes diferem entre si pelo teste Tukey $(\mathrm{P} \leq 0,05)$.

$\mathrm{RV}=$ dieta elaborada somente com ingredientes de origem vegetal; $\mathrm{RV}+\mathrm{A}=$ dieta elaborada com ingredientes de origem vegetal e ingredientes de origem animal.

${ }^{1} \mathrm{Y}=126,74+125,90 \mathrm{x}-32,76 \mathrm{x}^{2}\left(\mathrm{R}^{2}=0,70\right) .{ }^{2} \mathrm{Y}=786,74+27,68 \mathrm{x}-11,24 \mathrm{x}^{2}\left(\mathrm{R}^{2}=0,34\right) .{ }^{3} \mathrm{Y}=108,74+25,78 \mathrm{x}-$ $17,64 \mathrm{x}^{2}\left(\mathrm{R}^{2}=0,68\right) .{ }^{4} \mathrm{Y}=7,24-1,44 \mathrm{x}+1,14 \mathrm{x}^{2}\left(\mathrm{R}^{2}=0,81\right) .{ }^{5} \mathrm{Y}=584,76+48,77 \mathrm{x}-16,91 \mathrm{x}^{2}\left(\mathrm{R}^{2}=0,41\right) .{ }^{6} \mathrm{Y}=85,35+$ $18,09 \mathrm{x}-6,34 \mathrm{x}^{2}\left(\mathrm{R}^{2}=0,38\right) .{ }^{7} \mathrm{Y}=6,83-0,65+0,21 \mathrm{x}^{2}\left(\mathrm{R}^{2}=0,34\right)$.

\section{CONCLUSÕES}

A suplementação de glutamina na dieta proporciona melhor conversão alimentar na fase pré-inicial. A adição de L-glutamina na dieta para fase inicial melhora o desenvolvimento do intestino delgado de pintos de corte e proporciona melhor qualidade intestinal de pintos alimentados com dietas contendo produtos de origem animal.

\section{REFERÊNCIAS}

ALLEN, P.C. Production of free radical species during Eimeria maxima infections in chickens. Poultry Scie., v.63, p.1457-1463, 1997.

ANDRADE. M.A. Inoculação de Salmonella enterica subespecie enterica sorovar enteritidis fagotipo 4 em ovos embrionados de duas linhagens de frango de corte. Goiânia, 2005. 113f. Tese (Doutorado em Ciência Animal). Universidade Federal de Goiás, 2005.

ASSOCIATION of Official Analytical Chemists - AOAC. Official methods of analysis. 15. ed., v.1. Virginia: Arlington, 1990. 1117p.
BRUGALLI, I. Variação de aminoácidos nos ingredientes - importância prática e aplicação do NIRS para controlar a variabilidade. In: SIMPÓSIO SOBRE INGREDIENTES NA ALIMENTAÇÃO ANIMAL, 2002, Campinas. Anais... Campinas: CBNA, 2002. p.277-284.

BUTOLO, J.E. Qualidade de ingredientes na alimentação animal. Campinas: J.E. Butolo, 2002. 430p.

DVORIN, A.; ZOREF, Z.; MOKADY, S. et al. Nutritional aspects of hydrogenated and regular soybean oil added to diets of broiler chickens. Poultry Scie., v.77, p.820-825, 1998.

INOUE, Y.; GRANT, J.P.; SNYDER, P.J. Effect of glutamine supplemented intravenous nutrition on survival after $E$ coli induced peritonitis. $J$. Par. Ent. N., v.17, p.41-46, 1993.

ITO, N.M.K.; MIYAJI, C.I.; LIMA, E.A.; OKABAYSHI, S. Saúde gastrointestinal, manejo e medidas para controlar as enfermidades gastrointestinais. In: MENDES, A.A.; NAAS, I.A.; MACARI, M. Produção de frangos de corte. Campinas: FACTA, 2004. Cap. 13, p.205251. 
LODDI, M.M.; CARVALHO, T.B.; DIGNER, C. et al. Morfometria do intestino delgado de frangos de corte desafiados com Salmonella e suplementados com aditivos. Rev. Bras. C. Av., Supl. 8, p.178, 2006.

LOPES, K.L.A.M. Suplementação de glutamina em dietas de frangos de corte. 2008. 77f. Tese (Doutorado em Ciência animal) - Escola de Veterinária, Universidade Federal de Goiás, Goiânia.

LUNA, L.G. Manual of Histologic Staining Methods of the Armed Forces Institute of Pathology. 3th ed. New York: McGraw-Hill, 1968. 258p.

MACARI, M.; MAIORKA, A. Função gastrointestinal e seu impacto no rendimento avícola. In: CONFERÊNCIA APINCO DE CIÊNCIA E TECNOLOGIA AVÍCOLAS, 2000, Campinas. Anais... Campinas: FACTA, 2000. v.2, p.162-174.

MAIORKA, A.; SILVA, A.V.F.; SANTIM, E. et al. Influência da suplementação de glutamina sobre o desempenho e o desenvolvimento de vilos e criptas do intestino delgado de frangos de corte. Arq. Bras. Med. Vet. Zootec, v.52, 2000.

NASCIMENTO, G.M. Suplementação da LGlutamina em ração sem agente anticoccidiano para frangos: Desempenho e Desenvolvimento da mucosa intestinal. 2010. 40f. Dissertação (Mestrado em Ciência Animal). Escola de Veterinária e Zootecnia, Universidade Federal de Goiás, Goiânia, 2010.

NUNES, R.V.; ROSTAGNO, H.S.; NUNES, C.G. et al. Valores energéticos e coeficiente de metabilizabilidade de subprodutos de abatedouro avícola determinados com frangos em crescimento. Rev. Bras. C. Av., Supl. 8, p.96, 2006.

RIBEIRO, S.R.; JÚNIOR, P.E.P.; MIRANDA, A.C. et al Weight loss and morphometric study of intestinal mucosa in rats after massive intestinal resection. Influence of a glutamineenriched diet. Rev. Hosp. Cl. Fac. Med. de São Paulo, v.59, p.349-356, 2004.
ROSTAGNO, H.S.; ALBINO, L.F.T.; DONZELE, J.L. et al. Tabelas brasileiras para aves e suínos: composição de alimentos $e$ exigências nutricionais. Viçosa: UFV, 2005. $141 \mathrm{p}$.

SAKAMOTO, M.I. Desempenho, desenvolvimento $e$ atividade enzimática da mucosa intestinal de frangos de corte alimentados com dietas suplementadas com glutamina e nucleotídeos. 2009. 117f. Tese (Doutorado em Zootecnia). Faculdade de Zootecnia e Engenharia de Alimentos, Universidade de São Paulo, Pirassununga. 2009.

STATISTICAL Analysis system. SAS Institute Inc., SAS/STAT. User's guide. Version 6.11. 4. ed., v.2. Cary: SAS Institute Inc., 2000. 842 p.

WISEMAN, J.; SALVADOR, F. The influence of free fatty acid content and degree ofsaturation on the apparent metabolizable energy value of fats fed to broilers. Poult. Scie., v.70, p.573-582, 1991.

WU, G.; MEIER, S.A.; KNABE, A. Dietary glutamine supplementation prevents jejunal atrophy in weaned pigs. J. Nutr., v.126, p.25782584, 1996.

XAVIER, S.A.G.; STRINGHINI, J.H.; BRITO, A.B. et al. Desempenho de frangos de corte consumindo dietas com diferentes fontes protéicas na fase pré-inicial. Rev. Bras. C. Av., v.6, p.52, 2004.

YI, G.F.; CARROLL, J.A.; ALLEE, G.L. et al. Effect of glutamine and spray-dried plasma on growth performance, small intestinal morphology, and immune responses of Escherichia coli $\mathrm{K} 88^{+}$challenged weaned pigs. J. An. Sci., v.83, p.634-643, 2005.

ZAVARIZE, K.C.; SARTORI, J.R.; PELÍCIA, V.C. et al. Glutamina e nucleotídeos na dieta de frangos de corte criados no sistema alternativo. Arch. Zootec., v.60, p.380-395, 2011.

ZOLLITSCH, W.; KNAUS, W.; AICHINGER, F.; LETTNER, F. Effects of different dietary fat sources on performance and carcass characteristics of broilers. An. F. Sci. Tech., v.66, p.63-73, 1997. 